\title{
Downregulation of AC061961.2, LING01-AS1, and RP11-13E1.5 is associated with dilated cardiomyopathy progression
}

\author{
Zhibing Qiu ${ }^{1, *}$, Bin Ye ${ }^{2, *}$, Li Yin ${ }^{1}$, Wen Chen ${ }^{1}$, Yueyue $X_{u^{1}}$ and Xin Chen ${ }^{1}$ \\ ${ }^{1}$ Department of Thoracic and Cardiovascular Surgery, Nanjing First Hospital, Nanjing Medical University, Nanjing 210006, \\ Jiangsu Province, China \\ ${ }^{2}$ Department of Anesthesiology, Yangzhou Maternal and Child Health Hospital, Yangzhou 225002, Jiangsu Province, China \\ *These authors have contributed equally to this work \\ Correspondence to: Xin Chen, email: chenxinCX66@163.com \\ Keywords: dilated cardiomyopathy; differentially expressed gene; IncRNA; Weighted Gene Coexpression Network Analysis \\ Abbreviations: DCM: dilated cardiomyopathy; DEGs: differentially expressed genes; DELs: DE IncRNAs; WGCNA: Weighted Gene \\ Coexpression Network Analysis \\ Received: September 30, 2017 \\ Accepted: December 05, 2017 \\ Published: January 02, 2018 \\ Copyright: Qiu et al. This is an open-access article distributed under the terms of the Creative Commons Attribution License 3.0 \\ (CC BY 3.0), which permits unrestricted use, distribution, and reproduction in any medium, provided the original author and source \\ are credited.
}

\section{ABSTRACT}

Purpose: This study aimed to explore IncRNAs implicated in dilated cardiomyopathy (DCM) using high-throughput sequencing.

Methods: From February 2016 to May 2017, ten samples of failing hearts collected from the left ventricles of DCM patients undergoing heart transplants, and ten control samples obtained from normal heart donors were included in this study. After sequencing, raw data were processed, and differentially expressed genes (DEGs) and IncRNAs between samples from patients with DCM and controls were screened, followed by functional enrichment analysis and Weighted Gene Coexpression Network Analysis (WGCNA). Five key IncNRAs were validated through real-time PCR.

Results: After processing sequence data, 1398 DEGs were identified between DCM and control groups, including 267 IncRNAs. WGCNA identified seven modules that were significantly correlated with DCM. The top 50 genes in the three modules (black, dark-green, and green-yellow) were significantly correlated with the DCM disease state. Four core enrichment IncRNAs, such as AC061961.2, LINGO1-AS1, and RP11-557H15.4, in the green-yellow module were associated with functions of neurotransmitter secretion. Five core enrichment IncRNAs, such as KB-1299A7.2 and RP11-13E1.5, in the black module co-regulated functions associated with the regulation of blood circulation and heart contraction. AC061961.2, LINGO1-AS1, and RP11-13E1.5 were confirmed to be downregulated in DCM tissues by real-time PCR.

Conclusion: The present study suggests that downregulation of AC061961.2, LINGO1-AS1, and RP11-13E1.5 is associated with DCM progression, and these may serve as key diagnostic biomarkers and therapeutic targets for DCM.

\section{INTRODUCTION}

Cardiomyopathies are a group of heart muscle diseases characterized by abnormal chamber size and wall thickness or abnormal contractile function [1]. Dilated cardiomyopathy (DCM) is the most common cardiomyopathy worldwide, characterized by enlargement in left ventricular cavity, as well as impaired contractility in the absence of significant coronary artery disease [2]. Additionally, some abnormal loading conditions, including primary valvular diseases, congenital heart diseases and arterial hypertension may also result in DCM. DCM may lead to sudden cardiac death and progressive heart failure and thus, is related to serious morbidity and premature mortality [3]. Despite therapeutic advances, the 5-year mortality for DCM remains up to $20 \%$ [4]. Studies have found that genetic etiologies are 
associated with the pathogenesis of DCM $[5,6]$. Therefore, understanding the genetic basis of DCM and identifying causative genes in individuals with DCM may help in improving the clinical management of this disease.

Lakdawala et al. [6] recently studied a diverse population of patients with DCM for clinical genetic testing. They identified relevant genetic mutations in $17.4 \%$ of those DCM patients, commonly involving the myosin heavy chain 7, lamin A/C, and troponin T2 genes. The completion of the Human Genome Project revealed that $98 \%$ of the genome consists of non-coding sequences, including microRNAs and long non-coding RNAs (lncRNAs). LncRNAs are transcribed RNA molecules longer than 200 nucleotides that actively participate in various physiological and pathological processes [7]. Several recent studies have identified many lncRNAs associated with diseased hearts [8-10]. However, there are still not enough studies on the functions of lncRNAs in DCM [11, 12].

In this study, we aimed to explore more lncRNAs implicated in DCM using high-throughput sequencing of lncRNAs in myocardial tissues collected from DCM patients and normal heart donors. Based on sequencing data, we screened differentially expressed genes (DEGs) and lncRNAs (DELs), followed by a series of bioinformatic analyses for identifying key lncRNAs involved in DCM. The findings were then validated through RT-PCR.

\section{RESULTS}

\section{Raw read quality control and reference genome alignment}

The number of raw reads in paired ends of each sample was more than 30,000,000 (data not shown). After filtration, numbers of clean reads ranged from 28,248,054 to $35,511,833$ in each sample. The rates of clean reads were higher than $86.2 \%$. The rates of read alignment to the human reference genome (hg19) were between $83.1 \%$ and $85.8 \%$ in the DCM group and between $69.3 \%$ and $85.3 \%$ in the control group (data not shown).

\section{Differential gene and IncRNA analysis}

A total of 21,065 genes were expressed in all samples of at least one group (DCM or control). Among these genes, 4328 were $\operatorname{lncRNAs}$. With thresholds of $\mid \log$ (fold change) $\mid>$ 1 and adjusted p-value $<0.05,1398$ DEGs (447 upregulated and 951 downregulated ones) were identified. Additionally, these DEGs included 267 DELs (93 upregulated and 174 downregulated ones). Heat maps of DEGs and DELs are shown in Figure 1. DEGs and DELs could clearly distinguish between the DCM and control groups.

\section{Functional enrichment analysis}

Upregulated DEGs were significantly associated with biological processes linked to heart and muscle contraction as well as the pathway of cardiac muscle contraction (hsa04260) (Figure 2A). Downregulated DEGs were significantly connected to the following GO terms: regulation of membrane potential (GO:0042391), synapse organization (GO:0050808), and signal release (GO:0023061) as well as pathways of protein digestion and absorption (hsa04974), circadian entrainment (hsa04713), and calcium signaling pathway (hsa04020) (Figure 2B).

\section{PPI network and subnetwork module analysis}

The PPI network consisted of 3565 edges and 799 nodes (252 upregulated and 547 downregulated genes). The top 10 gene nodes with high degrees included $A L B$, TNF, SRC, JUN, RIPK4, FOS, MYC, ACTA1, ACTA2, and $E D N 1$. In addition, 21 modules were extracted from the PPI network. The two modules containing the most nodes are shown in Figure 3A and 3B. Module 1 contained 35 nodes and 287 edges, while module 2 included 36 nodes and 279 edges. DEGs in module 1 were significantly involved in neuroactive ligand-receptor interaction, phospholipase D signaling pathway, and calcium signaling pathway and DEGs in module 2 were particularly associated with the AGE-RAGE, TNF, and IL-17 signaling pathways (Figure 3C).

\section{WGCNA analysis}

DEGs were subjected to WGCNA module analysis, and each module contained at least 10 genes. A total of 13 modules were identified. Correlations between WGCNA modules and DCM are shown in Table 1. Upon applying the thresholds of p-value $<0.01$ and correlation coefficient $>0.7$, seven modules (indicated by different colors) were correlated with DCM, among which the magenta, blue, and purple ones were positively correlated with DCM, while the green-yellow, salon, dark-green, and black ones were negatively correlated with it. In this study, we focused on the top 50 genes in these seven modules. LncRNAs were included among the top 50 genes in each module.

\section{Correlation analysis of WGCNA modules and the DCM disease state}

Using GSAASeqSP, correlations between the top 50 genes in the seven modules and the DCM disease state were analyzed. The correlation p-values between three modules (black, dark-green, and green-yellow) and the DCM disease state were less than 0.05. These three modules are shown in Figure 4. After GASS analysis, the core enrichment genes in these three modules were obtained. The black module had 35 core enrichment genes, which included five lncRNAs (AC009878.2, CTB-174D11.1/ KB-1299A7.2, RP11-557H15.4, and RP4-534N18.2); the dark-green module had 12 core enrichment genes without 
any lncRNAs; and the green-yellow module had 31 core enrichment genes, including four lncRNAs (AC061961.2, LINGO1-AS1, RP11-13E1.5, and RP11-218M11.3).

\section{IncRNA regulatory network analysis}

The mRNAs involved in the three above-mentioned modules were regarded as lncRNA correlated genes. Based on the nine lncRNAs identified in the three modules, we constructed lncRNA regulatory networks, as shown in Figure 5.

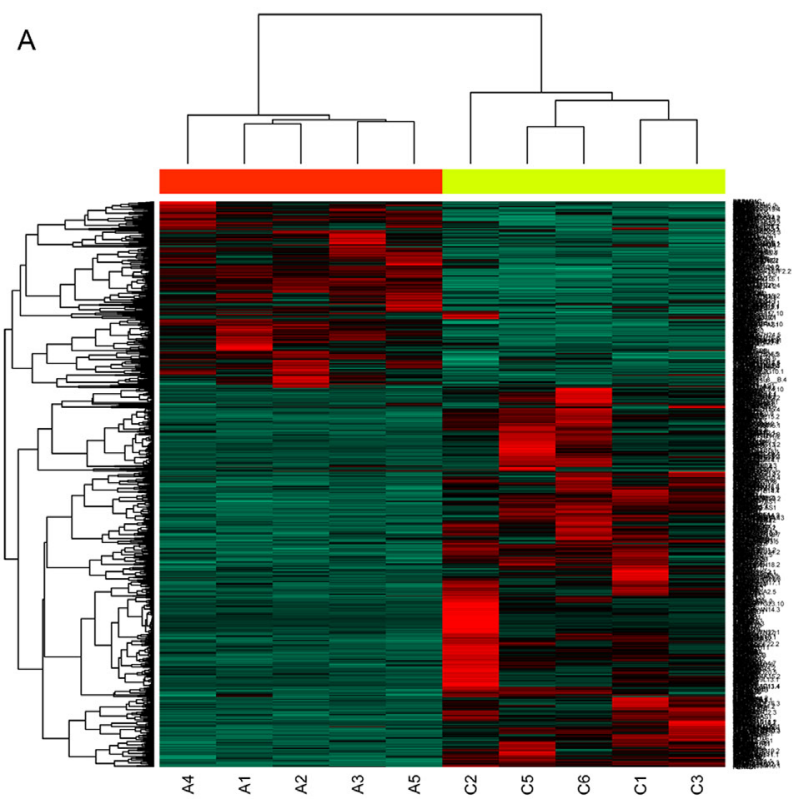

\section{Functional enrichment analysis of IncRNAs}

These lncRNA correlated genes were subjected to functional enrichment analysis. As shown in Figure 6, the five core enrichment lncRNAs in the black module co-regulated functions associated with regulation of blood circulation, heart contraction, membrane potential, heart process, heart contraction, and muscle contraction. Meanwhile, in the green-yellow module, the four core enrichment lncRNAs co-regulated functions of synaptic vesicle exocytosis, regulation of calcium ion-dependent

B

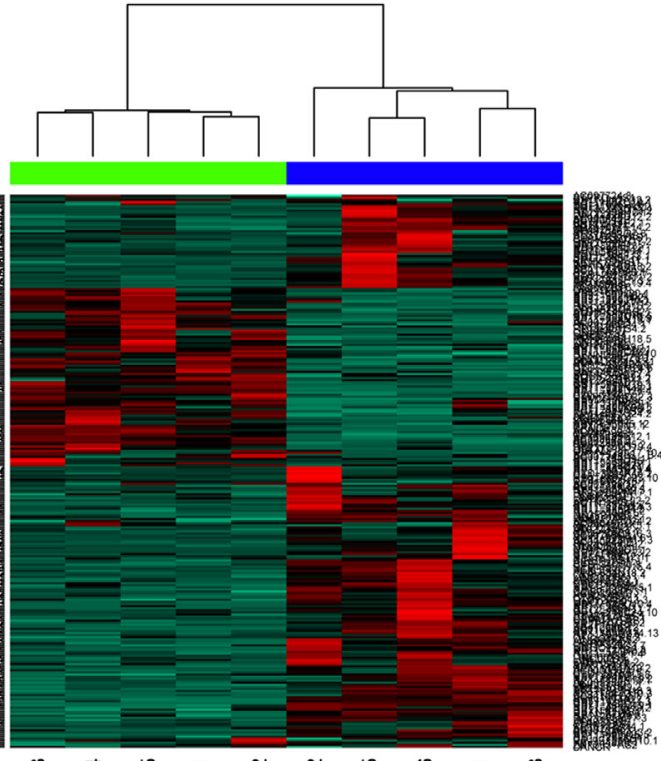

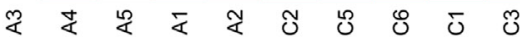

Figure 1: The heat maps of (A) differentially expressed genes and (B) differentially expressed lncRNAs between dilated cardiomyopathy (DCM) and control samples.

A
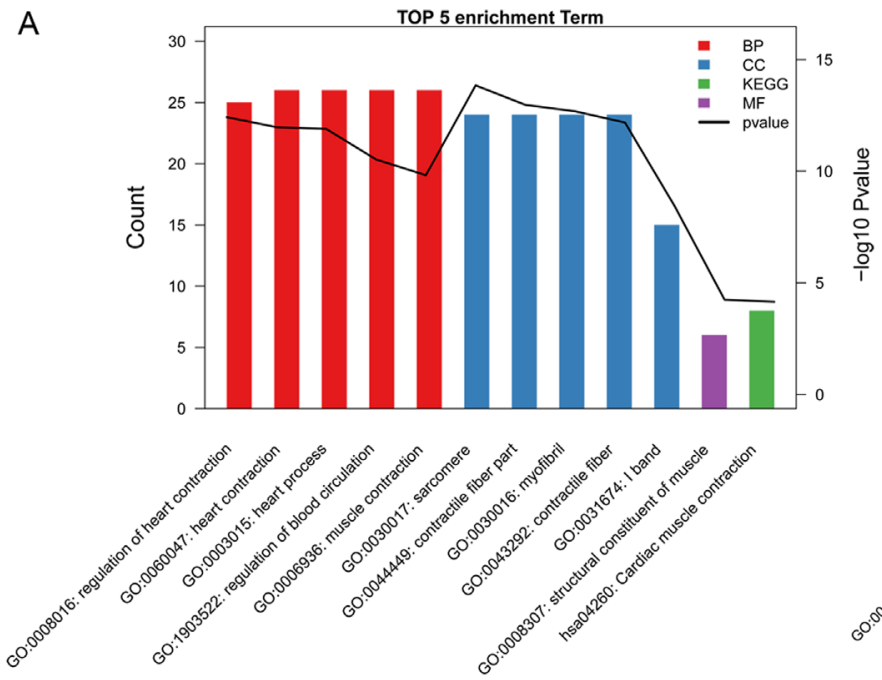

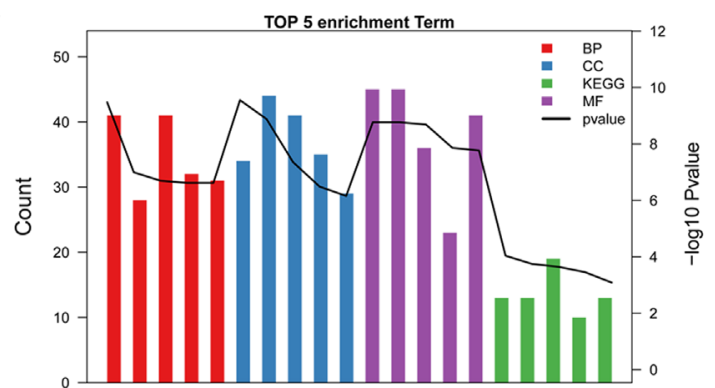

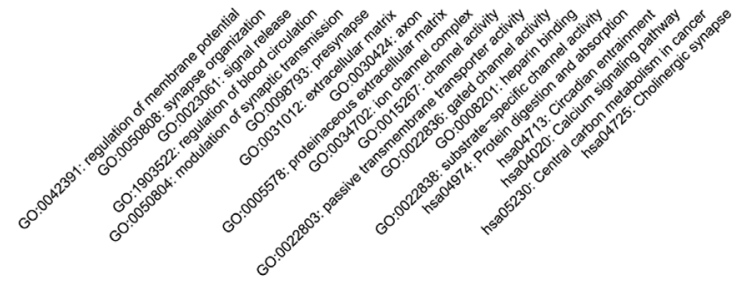

Figure 2: Gene Ontology (GO) functions and Kyoto Encyclopedia of Genes and Genomes (KEGG) pathways enriched by (A) upregualted and (B) downregulated differentially expressed genes. The x-axis represents GO and pathway terms, and the y-axis represents the number of genes enriched in terms. BP: biological process; CC: cellular component; MF: molecular function. 
exocytosis, establishment of synaptic vesicle localization, and neurotransmitter secretion.

\section{Potential drug small molecules for treating DCM}

With a p-value of $<0.05,37$ drug small molecules were predicted by correlated genes of AC009878.2, CTB-174D11.1, KB-1299A7.2, RP11-557H15.4, and RP4-534N18.2 (black module), such as N-[4-(3-oxo-3phenylprop-1-en-1-yl)phenyl]-2,2-diphenylacetamide, 4,5-dianilinophthalimide, and podophyllotoxin. Among these molecules, 15 had functions of inhibiting DCM progression (enrichment score $>0$ ), whereas 22 had functions of promoting it (enrichment score $<0$ ) (Table 2).
In addition, lncRNAs correlated genes in the greenyellow module (AC061961.2, LINGO1-AS1, RP1113E1.5, and RP11-218M11.3) predicted 82 potential drug small molecules, including 42 inhibiting DCM and 40 promoting it (Table 2).

\section{Real-time PCR verification of the expression of key IncRNAs}

Expression levels of the top five key lncRNAs with low p-values, namely, AC061961.2, LINGO1-AS1, RP11-557H15.4, KB-1299A7.2, and RP11-13E1.5, were determined using real-time PCR. As shown in Figure 7, AC061961.2, LINGO1-AS1, and RP11-13E1.5 were
A

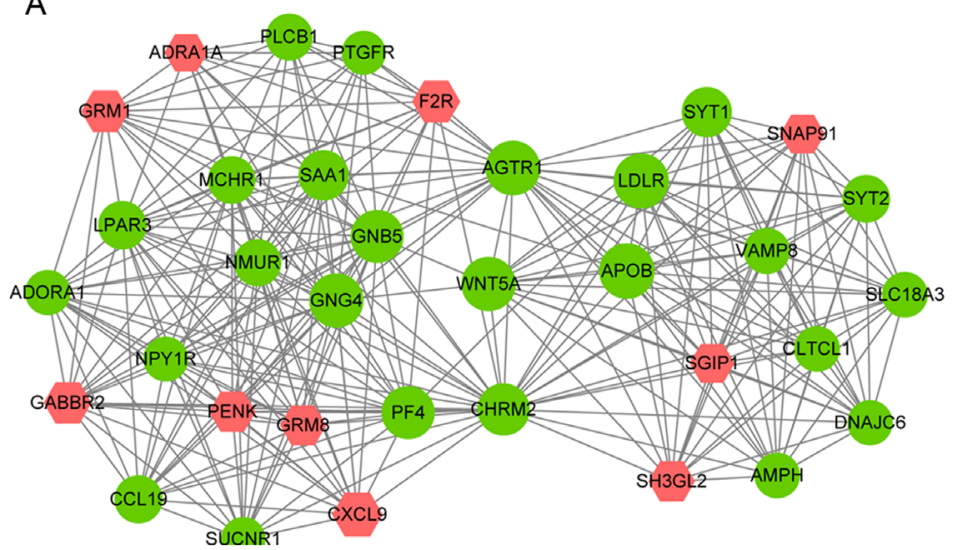

B

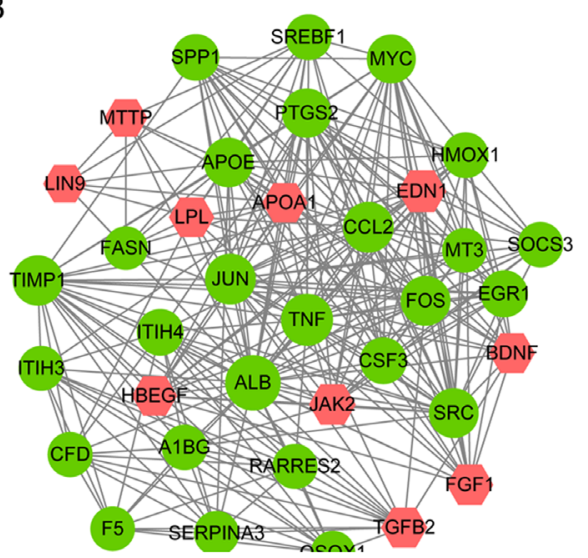

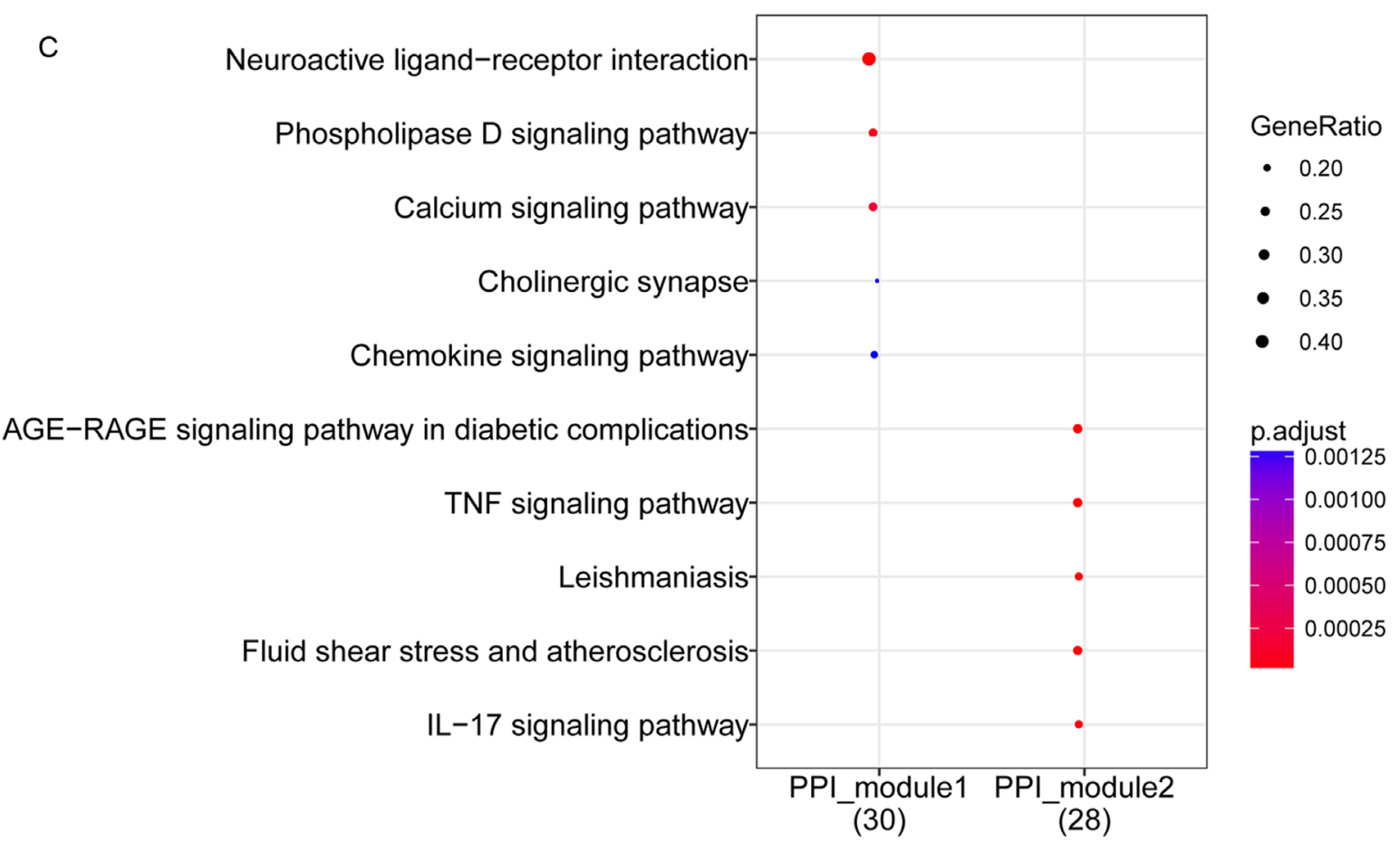

Figure 3: The top 2 protein-protein interaction modules containing more nodes. (A) Module 1 contains 35 nodes and 287 edges. (B) Module 2 includes 36 nodes and 279 edges. Red hexagon represents upregualted genes and green circle represents downregualted genes. (C) Kyoto Encyclopedia of Genes and Genomes (KEGG) pathways enriched by differentially expressed genes in module 1 and module 2 . 
Table 1: The correlations between Weighted Gene Co-expression Network Analysis (WGCNA) modules and dilated cardiomyopathy

\begin{tabular}{|c|c|c|c|c|c|c|c|c|c|c|c|c|c|}
\hline Module & Darkturquoise & Magenta & Lightyellow & Blue & Purple & Greenyellow & Royalblue & Salmon & Darkgreen & Turquoise & Black & Lightcyan & Grey \\
\hline Cor & 0.71 & 0.79 & 0.73 & 0.96 & 0.85 & -0.88 & -0.63 & -0.85 & -0.8 & -0.58 & -0.88 & -0.67 & 0.58 \\
\hline$P$ value & $2.20 \mathrm{E}-02$ & $6.40 \mathrm{E}-03$ & $1.62 \mathrm{E}-02$ & $1.39 \mathrm{E}-05$ & $1.72 \mathrm{E}-03$ & $6.83 \mathrm{E}-04$ & 5.07 E-02 & 2.04 E-03 & $5.16 \mathrm{E}-03$ & 7.59 E-02 & 8.69 E-04 & 3.41 E-02 & 8.16 E-02 \\
\hline
\end{tabular}

Cor: correlation coefficient.
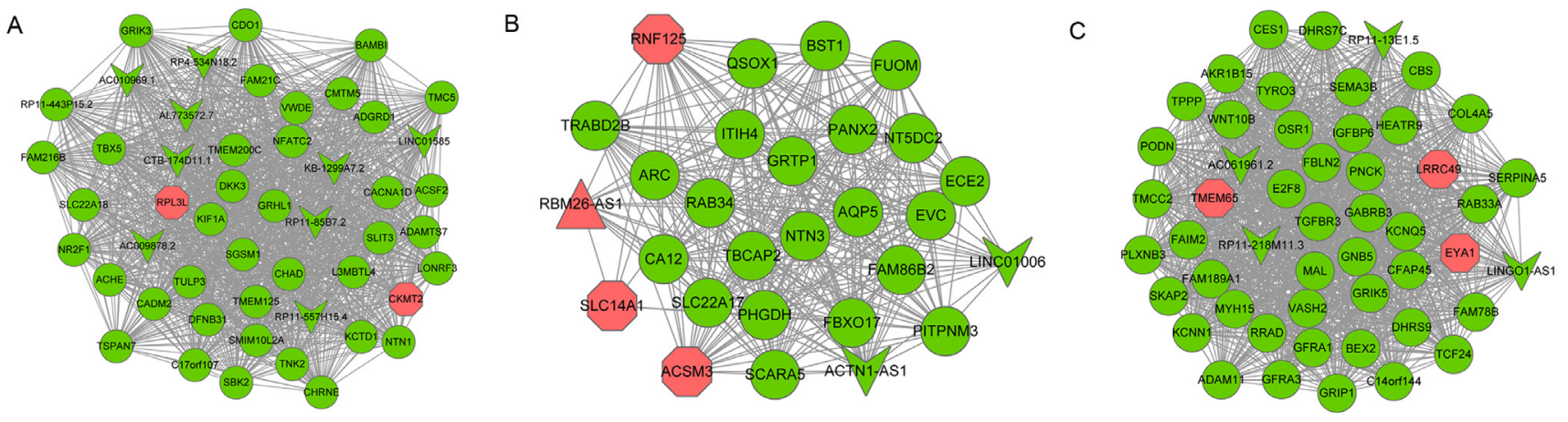

Figure 4: The network of top 50 genes in (A) black, (B) darkgreen and (C) greenyellow Weighted Gene Co-expression Network Analysis (WGCNA) modules. Red hexagon represents upregualted genes; green circle represents downregualted genes; red triangle represents upregualted lncRNA; green V-type represents downregualted lncRNA.
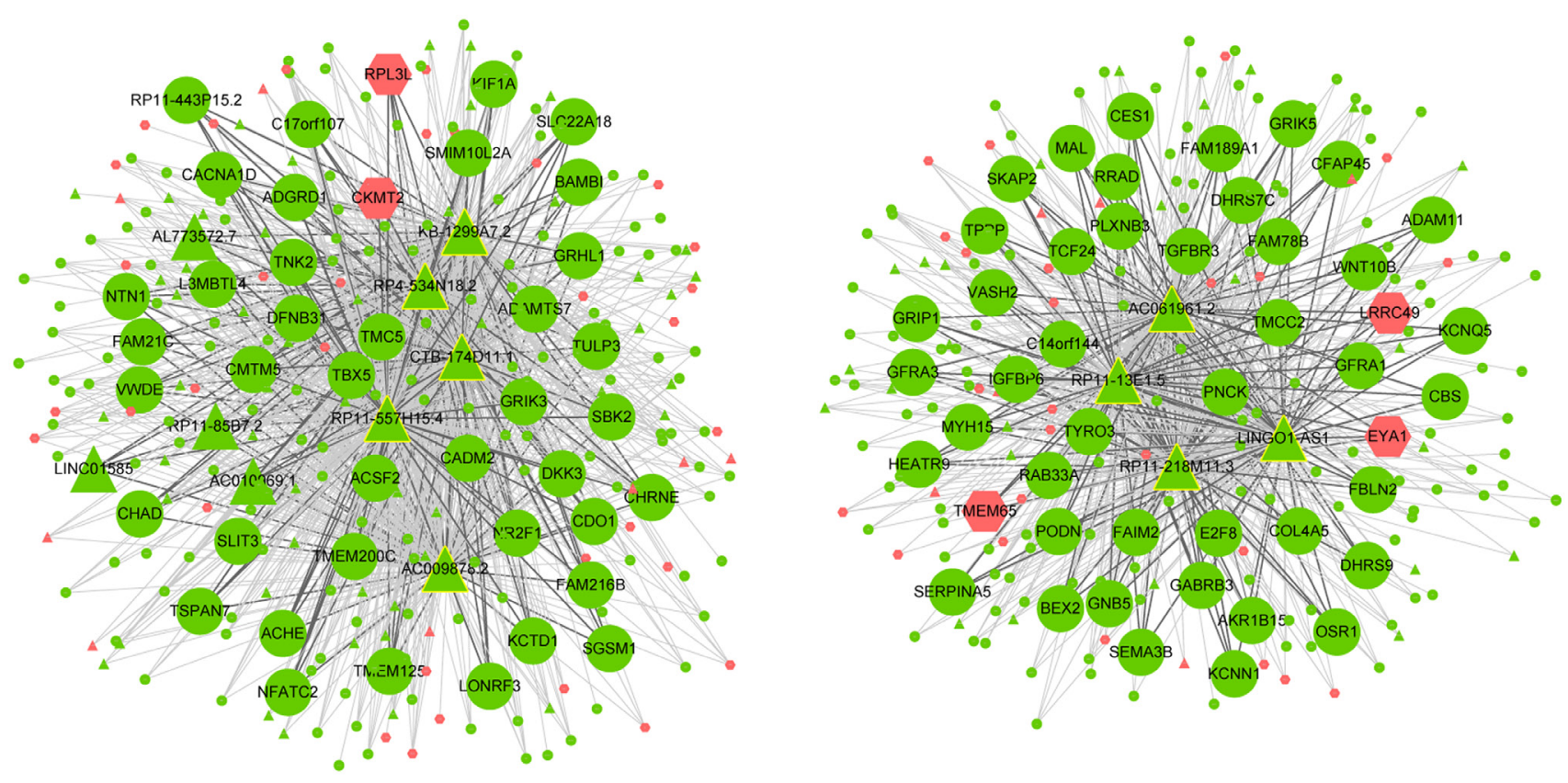

Figure 5: The IncRNA regulatory networks based on the nine IncRNAs and their correlated genes. Red hexagon represents upregualted genes; green circle represents downregualted genes; red triangle represents upregualted lncRNA; green triangle represents downregualted lncRNA. 


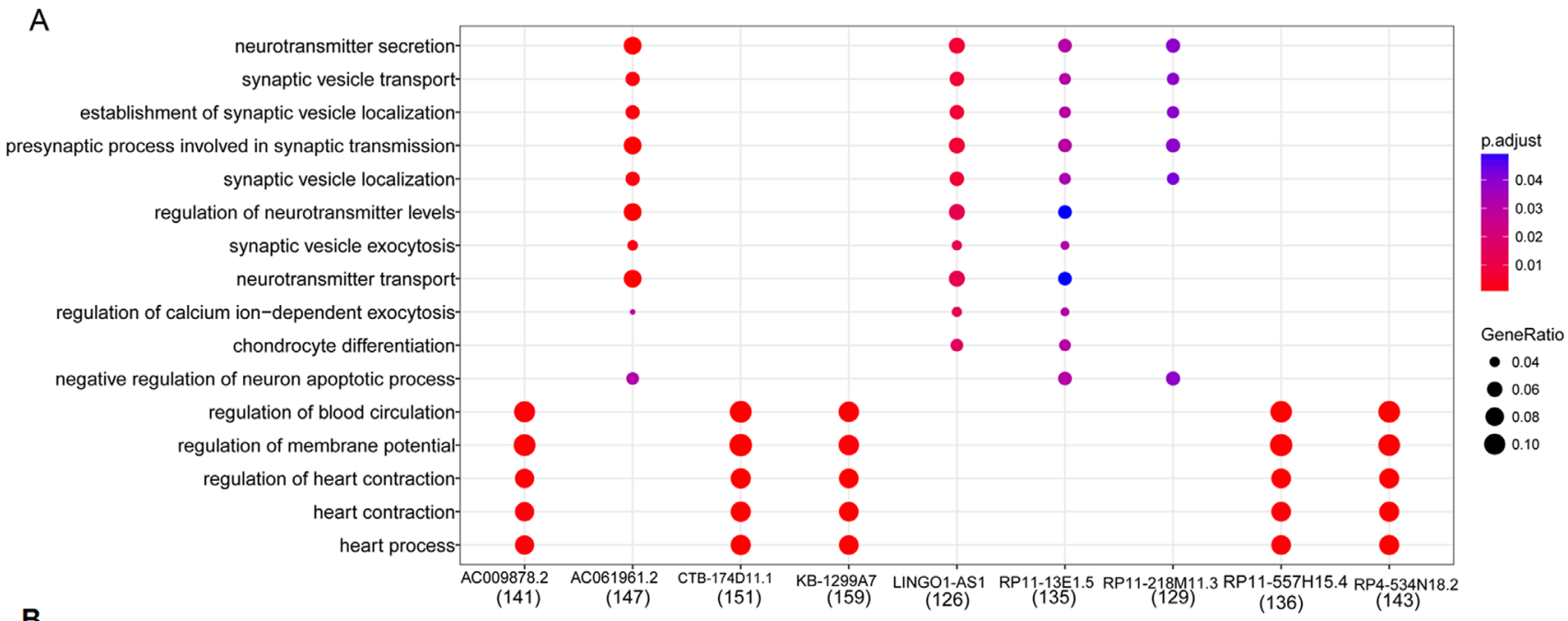

B

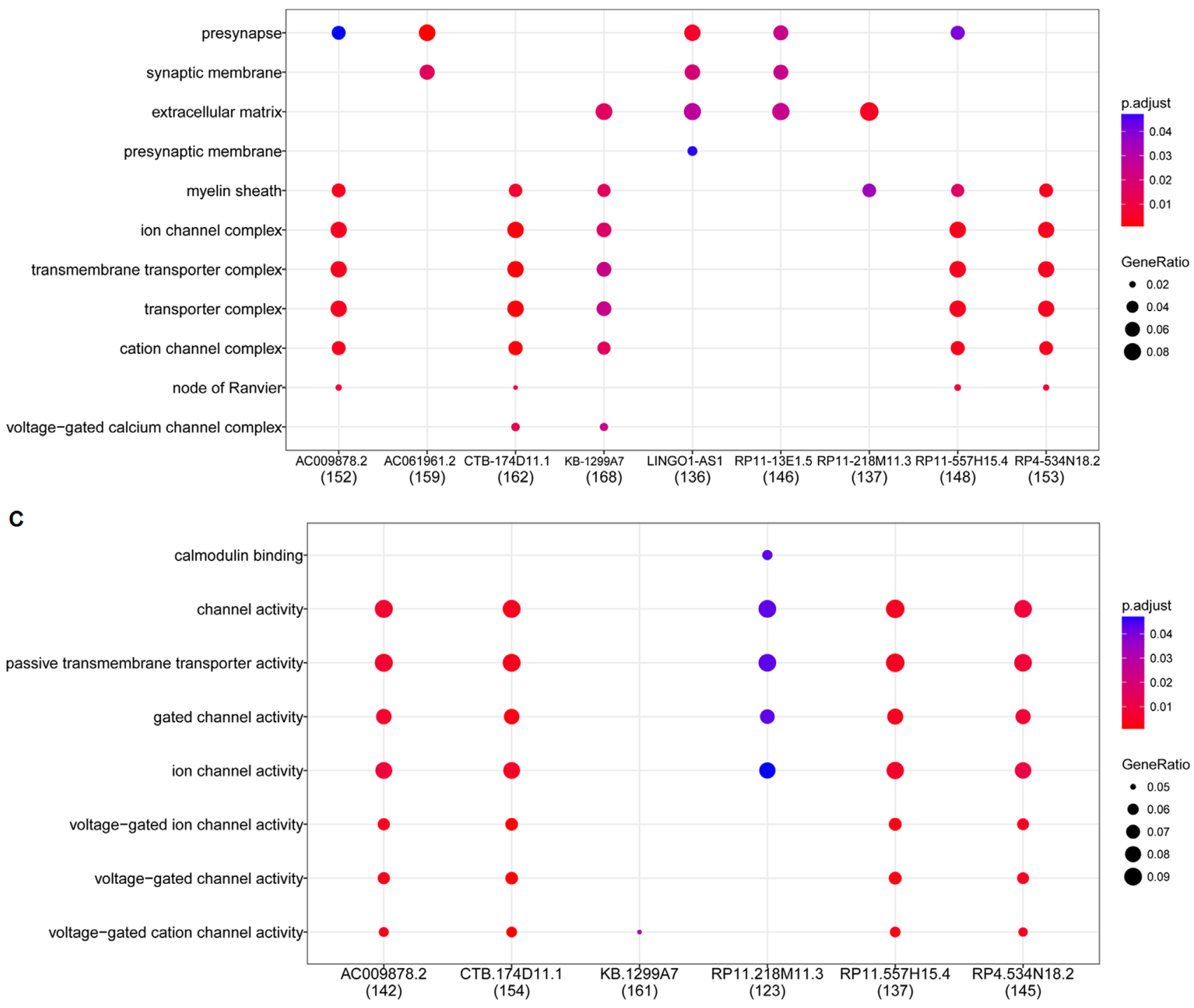

Figure 6: The Gene Ontology (GO) functions of (A) biological process, (B) cellular component and (C) molecular function enriched by the correlated genes of the nine lncRNAs. 
Table 2: Top 10 (absolute value of enrichment score) potential drug small molecules treating dilated cardiomyopathy

\begin{tabular}{lcc}
\hline Small molecules name & Enrichment score & P value \\
\hline Small molecules of AC009878.2/CTB-174D11.1/KB-1299A7.2/RP11-557H15.4/RP4-534N18.2 & \\
5224221 & 0.919 & 0.01312 \\
4,5-dianilinophthalimide & 0.881 & 0.02855 \\
prednicarbate & 0.868 & 0.00423 \\
5279552 & 0.846 & 0.04801 \\
ethionamide & 0.747 & 0.03187 \\
podophyllotoxin & -0.765 & 0.00629 \\
Amrinone & -0.781 & 0.00475 \\
0225151-0000 & -0.781 & 0.02149 \\
anisomycin & -0.836 & 0.00127 \\
docosahexaenoic acid ethyl ester & -0.894 & 0.02235 \\
Small molecules of AC061961.2/LINGO1-AS1/RP11-13E1.5/RP11-218M11.3 & \\
1,4-chrysenequinone & 0.848 & 0.04672 \\
natamycin & 0.761 & 0.00625 \\
0297417-0002B & 0.751 & 0.03067 \\
STOCK1N-28457 & 0.75 & 0.03099 \\
Ouabain & 0.74 & 0.00875 \\
Prestwick-972 & -0.814 & 0.01278 \\
Timolol & -0.82 & 0.00197 \\
ikarugamycin & -0.858 & 0.00565 \\
tribenoside & -0.924 & 0.00004 \\
thapsigargin & -0.94 & 0.00032 \\
\hline Enichmt & & \\
\hline
\end{tabular}

Enrichment score: a threshold value between +1 and -1 reflecting the correlation between drug small molecules and genes. 5224221: N-[4-(3-oxo-3-phenylprop-1-en-1-yl)phenyl]-2,2-diphenylacetamide; 527955: 6-chloro-3-phenyl [1, 2, 4] triazolo[4,3-b]pyridazine.

significantly downregulated in samples from DCM patients compared with that in control samples $(\mathrm{p}<0.01)$. RP11-557H15.4 was downregulated in the DCM group, albeit without a significant difference. However, KB1299A7.2 was not detected in samples from patients with DCM, which may be due to its expression level being too low in them.

\section{DISCUSSION}

In the present study, we obtained 1398 DEGs from the comparison between DCM and control groups; these DEGs included 267 DELs. WGCNA of these DEGs identified seven modules significantly correlated with DCM. Moreover, the top 50 genes in the black, dark-green, and green-yellow modules were significantly correlated with the DCM disease state. Additionally, nine core enrichment lncRNAs, including AC061961.2, LINGO1AS1, RP11-557H15.4, KB-1299A7.2, and RP11-13E1.5, were obtained from the black and green-yellow modules. Expression levels of AC061961.2, LINGO1-AS1, and RP11-13E1.5 in tissue samples from patients with DCM were verified through real-time PCR.

LncRNAs have been implicated in specifc physiological and pathological processes, including cardiovascular diseases $[13,14]$. A recent study of Greco et al. [15] investigated lncRNAs deregulated in patients with non end-stage dilated hypokinetic ischemic cardiomyopathy. They identified 14 lncRNAs deregulated in these patients, such as CDKN2B-AS1/ANRIL, EGOT and H19. Additionally, Frade et al. [16] performed a whole-transcriptome analysis of heart biopsy specimens of DCM patients and healthy hearts donors and revealed that MIAT was overexpressed in patients with DCM. Whereas, 
these lncRNAs were not identified in our study, which may be due to the sample heterogeneity.

Cardiac neurotransmitter systems are impaired in heart diseases. In patients with heart failure, abnormalities in the cardiac neurotransmitter systems contribute to arrhythmogenesis and cardiac dysfunction [17]. Qin et al. [18] also found that the heightened sympathetic state during congestive heart failure was associated with decreased cardiac sympathetic neurotransmitters. The role of the cardiac neurotransmitter system in DCM has not been fully investigated. In the present study, AC061961.2, LINGO1-AS1, and RP11-13E1.5 were significantly associated with the functions of neurotransmitter secretion and transport. Additionally, they were downregulated in samples from patients with DCM, which suggest their roles in DCM progression.

LncRNA regulatory networks were constructed based on WGCNA modules, and SOX5 was predicted to be a correlated gene of AC061961.2, LINGO1-AS1, and RP11-13E1.5. SOX5 belongs to the SOX family of transcription factors, which is expressed in many human tissues, including the heart [19, 20]. Genome-wide association studies have implicated $S O X 5$ as a candidate gene for susceptibility to the electrocardiographic PR interval [21], higher resting heart rate [22], left ventricular mass [23], and atrial fibrillation [24], suggesting its importance in the heart. A recent study also revealed that SOX5 regulated cardiac function by negatively regulating Wnt signaling, which had been implicated as an important event regulating cardiac function and development [25]. Taking these findings together, these three lncRNAs may be involved in the progression of DCM by interacting with SOX5.

The correlated genes of lncRNAs in the black module co-regulated functions associated with the regulation of blood circulation, heart contraction, heart process, and membrane potential. For instance, ADORA1 was a correlated gene of RP11-557H15.4 and KB-1299A7.2, which was also involved in the PPI subnetwork module 1. ADORA1 was significantly enriched in the above GO functions. ADORA1 is a member of the adenosine receptor family of G-proteincoupled receptors with adenosine as an endogenous ligand [26]. Adenosine is implicated in cardioprotective events, particularly in cardiac hypertrophy [27]. For example, Xu et al. [28] reported that reduced adenosine levels results in
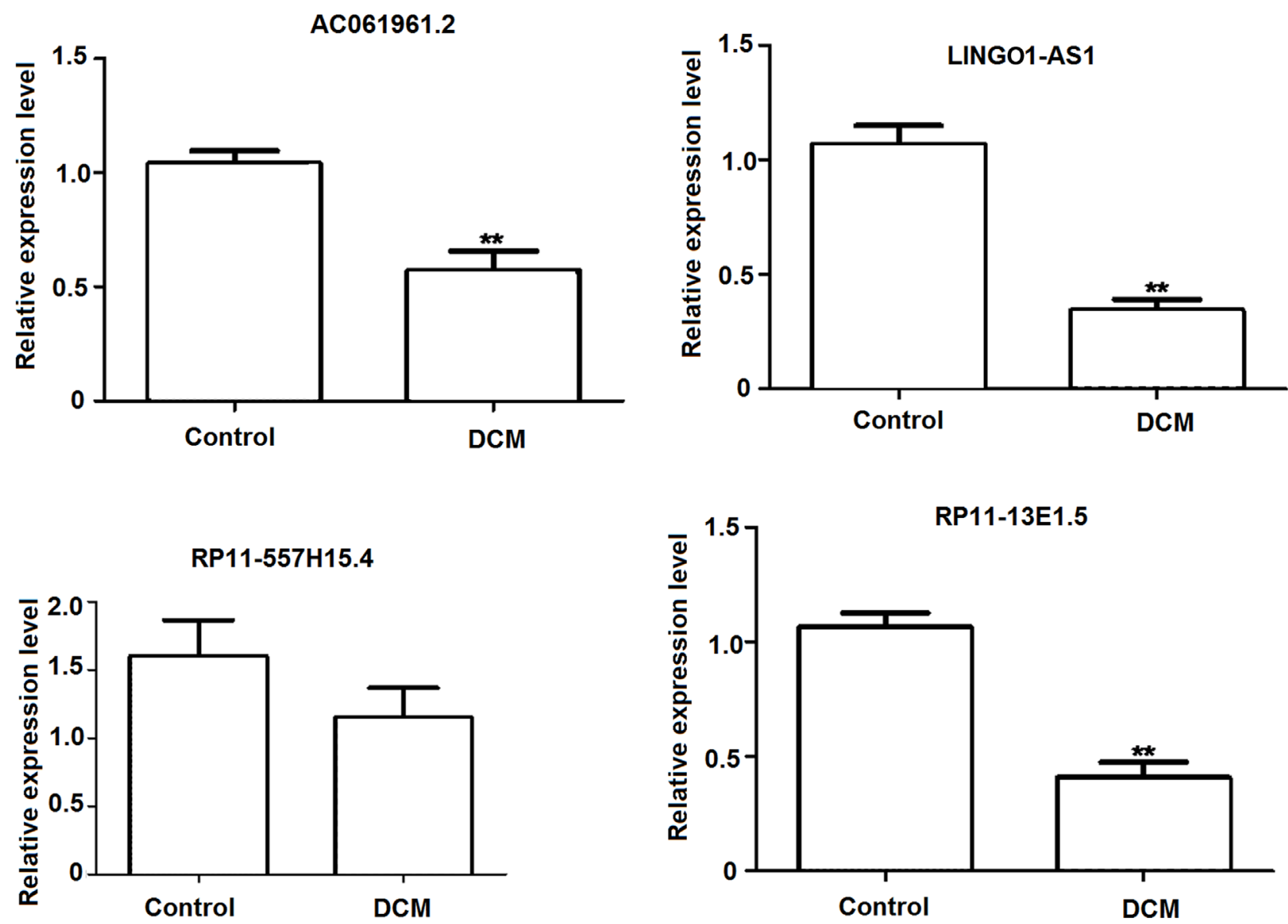

Figure 7: Relative expression levels of AC061961. 2, LINGO1-AS1, RP11-557H15.4 and RP11-13E1.5 determined by real time PCR. Their expression levels are normalized against GAPDH. ${ }^{*}, P<0.01$. 
Table 3: Characteristics of patients

\begin{tabular}{llcl}
\hline Samples & Sex & Age & Group \\
\hline A1 & Female & 38 & Idiopathic dilated cardiomyopathy \\
A2 & Male & 43 & Idiopathic dilated cardiomyopathy \\
A3 & Female & 51 & Idiopathic dilated cardiomyopathy \\
A4 & Male & 57 & Idiopathic dilated cardiomyopathy \\
A5 & Male & 45 & Idiopathic dilated cardiomyopathy \\
A6 & Female & 39 & Idiopathic dilated cardiomyopathy \\
A7 & Male & 44 & Idiopathic dilated cardiomyopathy \\
A8 & Male & 56 & Idiopathic dilated cardiomyopathy \\
A9 & Female & 59 & Idiopathic dilated cardiomyopathy \\
A10 & Male & 42 & Idiopathic dilated cardiomyopathy \\
C1 & Male & 42 & Control \\
C2 & Male & 52 & Control \\
C3 & Female & 54 & Control \\
C4 & Female & 48 & Control \\
C5 & Male & 60 & Control \\
C6 & Female & 51 & Control \\
C7 & Male & 40 & Control \\
C8 & Female & 47 & Control \\
C9 & Male & 56 & Control \\
C10 & Female & &
\end{tabular}

Table 4: The primers used in real time PCR

\begin{tabular}{ll}
\hline Primer name & Sequences (5'-3') \\
\hline AC061961.2-hF & ATGCTTCTCCTGCCAGTTCG \\
AC061961.2-hR & TTTCCACTTCCCACGGTGTCA \\
LINGO1-AS1-hF & TGCCTTTCAGACACATCCAAG \\
LINGO1-AS1-hR & TGTCACTACCACGTCTAGTCCA \\
RP11-557H15.4-hF & TTCCACCCCTTGATCCCCGTA \\
RP11-557H15.4-hR & CTTCTCGTGTTCCCCGTCACC \\
RP11-13E1.5-hF & GCCCATCTTGTTGGACTGCT \\
RP11-13E1.5-hR & CTTCTGGTGGCTCCTTGCAT \\
GAPDH-hF & TGACAACTTTGGTATCGTGGAAGG \\
GAPDH-hR & AGGCAGGGATGATGTTCTGGAGAG \\
\hline
\end{tabular}


obvious cardiac hypertrophy in rat model. A recent study also suggested that ADORA1 prevents transition from compensated myocardial hypertrophy to decompensated heart failure [29]. Therefore, RP11-557H15.4 and KB1299A7.2 may involve in DCM pregression through the downregulation of ADORA1.

Establishment of the relationship among diseases, physiological processes, and the action of smallmolecule therapeutics is a fundamental challenge arising throughout biomedicine. Connectivity maps are used to search for connections among small molecules sharing a mechanism of action, physiological processes and chemicals, and diseases and drugs [30]. In this study, we used a connectivity map for predicting potential drug small molecules for treating DCM. Many small molecules, such as N-[4-(3-oxo-3-phenylprop-1-en-1-yl)phenyl]2,2-diphenylacetamide, 4,5-dianilinophthalimide, and 1,4-chrysenequinone, were predicted, but their roles in DCM require further validation.

In conclusion, the present study suggests that AC061961.2, LINGO1-AS1, and RP11-13E1.5 are downregulated in myocardial tissues of patients with DCM. Additionally, downregulation of RP11-557H15.4 and KB-1299A7.2 may also be associated with DCM, although this requires further confirmation. These lncRNAs may serve as key diagnostic biomarkers and therapeutic targets for DCM.

\section{MATERIALS AND METHODS}

\section{Samples}

Ten human failing heart tissue samples were collected from the left ventricles of DCM patients who accepted heart transplants. These patients aged 38-59 (average age $47.4 \pm 2.4$ ) years and were enrolled from February 2016 to May 2017. The inclusion criterion was that they should be idiopathic DCM. The patients with secondary cardiomyopathy were excluded. Additionally, ten healthy heart samples (as control) were obtained from the left ventricles of normal heart donors (average age 51.2 \pm 2.3 years) that were unsuitable to transplant for several technical reasons. Detailed patient information is shown in Table 3. All patients provided informed consent before the study. All procedures in this study were approved by our hospital's protection of human ethics committee.

\section{Total RNA isolation and quality control}

Total RNAs were isolated from 10 myocardial tissue samples ( 5 samples from DCM group and 5 from control group) using a TRIzol reagent (Invitrogen, CA, USA). RNA integrity was assessed using the RNA Nano 6000 Assay Kit of the Agilent Bioanalyzer 2100 system (Agilent Technologies, CA, USA). NanoPhotometer ${ }^{\mathbb{R}}$ spectrophotometer (IMPLEN, CA, USA) was used to check RNA purity, and Qubit ${ }^{\mathbb{R}}$ RA Assay Kit in Qubit ${ }^{\mathbb{}} 2.0$ Flurometer (Life Technologies, CA, USA) was used to measure the RNA concentration.

\section{Library preparation}

For each sample, $3 \mu \mathrm{g}$ of RNA was used for the preparation of RNA samples. Ribosomal RNA was removed using the Epicentre Ribo-zeroTMrRNA Removal Kit (Epicentre, USA). rRNA-free residue was removed by ethanol precipitation. Sequencing libraries were then generated with the NEBNext ${ }^{\circledR}$ Ultra TM Directional RNA Library Prep Kit for Illumina ${ }^{\circledR}$ (NEB, USA). Briefly, fragmentation was performed using divalent cations in NEBNext First Strand Synthesis Reaction Buffer $(5 \times)$. First-strand cDNA was synthesized using M-MuLV Reverse Transcriptase and random hexamer primers, and second-strand cDNA was synthesized using RNase $\mathrm{H}$ and DNA Polymerase I. In the reaction buffer, dTTP of dNTPs was replaced by dUTP. After adenylation of the $3^{\prime}$ ends of the DNA fragments, NEBNext Adaptor with a hairpin loop structure was ligated to prepare for hybridization. To select cDNA fragments preferentially, $250-300 \mathrm{bp}$ in length, the library fragments were purified with the AMPure XP system (Beckman Coulter, MA, USA). Then, $3 \mu \mathrm{L}$ of USER Enzyme (NEB, USA) was used with sizeselected, and adaptor-ligated cDNA at $37^{\circ} \mathrm{C}$ for $15 \mathrm{~min}$ followed by 5 min at $95^{\circ} \mathrm{C}$. Next, PCR was performed based on Universal PCR primers, Index (X) Primer, and Phusion High-Fidelity DNA polymerase. The products were purified (AMPure XP system), and the library quality was assessed on the Agilent Bioanalyzer 2100 system.

\section{Clustering and sequencing}

Index-coded samples were clustered on a cBot Cluster Generation System using HiSeq4000 PE Cluster Kit (Illumina). Then libraries were sequenced on an Illumina Hiseq4000 platform and 150-bp paired-end reads were generated. Obtained sequencing data were deposited in the NCBI SRA database (http://www.ncbi.nlm.nih.gov/ sra) under series accession number SRP108571.

\section{Raw read quality control and reference genome alignment}

Obtained raw reads were quality-filtered to remove low-quality reads and adaptor sequences using Fastx toolkit version 0.0.13 and Prinseq-lite version 0.20.4 [31]. Obtained clean data were aligned to the human reference genome (hg19) using TopHat version 2.0.8 [32].

\section{Quantitative and differential gene and IncRNA analyses}

Based on the gene and lncRNA annotation information in the GENCODE version 24 (mapped to 
GRCh37) database [33], we obtained the fpkm value and read count of genes using the StringTie version 1.2.2 [34]. DEGs including DELs were identified between the disease and normal control groups using the edgeR [35] package in $\mathrm{R}$ by applying the following criteria: $\mid \log$ (fold change)| $>1$ and adjusted $p$-value $<0.05$.

\section{Prediction of IncRNA correlated genes}

Weighted Gene Coexpression Network Analysis (WGCNA) is used for identifying clusters (modules) of highly correlated genes [36]. In this study, all DEGs including DELs were subjected to WGCNA using the WGCNA package [36] in R. mRNAs in the module that contained DELs were regarded as correlated genes of that lncRNA.

\section{Functional enrichment analysis}

DEGs and DELs correlated genes were subjected to Gene Ontology (GO) and Kyoto Encyclopedia of Genes and Genomes pathway enrichment analyses using clusterprofiler [37] in R.

\section{Correlation analysis of WGCNA modules and DCM disease state}

The correlation between WGCNA modules and DCM was analyzed using one-way ANOVA and the module with a p-value of $<0.05$ was selected. Additionally, based on the expression values of module genes, we analyzed the correlation between module genes and the DCM disease state using a toolset of Gene Set Association Analysis for RNA-Seq with Sample Permutation (GSAASeqSP, http://gsaa.unc.edu) [38], and the module with a nominal p-value of $<0.05$ was considered to be significantly correlated with the DCM disease state. Furthermore, according to the core enrichment gene obtained from GSAASeqSP, we screened key lncRNAs and constructed the lncRNA regulatory network on the basis of the WGCNA module. The lncRNA regulatory network was then visualized through Cytoscape [39].

\section{Protein-protein interaction (PPI) network analysis}

PPI networks of DEGs and DELs correlated genes were predicted using the Search Tool for the Retrieval of Interacting Genes/Proteins (STRING version 10.0, http:// string-db.org) [40] and were visualized using Cytoscape [39]. In addition, PPI subnetwork modules were identified using the MCODE [41] plugin in Cytoscape. Parameters of MCODE were set as follows: degree cut-off $=5$, node score cut-off $=0.2$, and $\mathrm{K}$-Core $=2$.

\section{Prediction of potential drug small molecules for treating DCM}

For the selected key lncRNAs in WGCNA modules, we predicted their potential drug molecules from the connectivity map [42] database according to their expression levels.

\section{Real-time PCR verification of the expression of key IncRNAs}

A total of 20 samples, including 10 samples from patients with DCM and 10 from controls, were used for real-time PCR verification of the expression of key lncRNAs. Briefly, total RNAs were isolated using a TRIzol reagent (Invitrogen, CA, USA). RNA concentration and quality were determined on a TECAN infinite M100 PRO Biotek microplate reader (TECAN, CA, USA). Then 0.5 $\mu \mathrm{g}$ of the total RNA was used from cDNA synthesis using the PrimeScript RT Master Mix (RR036A; Takara, Dalian, China). PCR was performed using the SYBR GREEN kit (4367659; Thermo, USA) in the Viia7 Real-Time PCR System (Applied Biosystems, USA). The primers used in this study are listed in Table 4.

\section{Statistical analysis}

Data are presented as mean \pm standard deviation. Statistical analysis was performed using SPSS 22.0 (IBM, Armonk, NY, USA). Differences in miRNA expression levels among different groups were analyzed by one-way analysis of variance and were considered significant if $p$ $<0.05$.

\section{Author contributions}

Conception and design of the research: Zhibing Qiu and Xin Chen; Acquisition of data: Bin Ye and Li Yin; Analysis and interpretation of data: Bin Ye and Wen Chen; Statistical analysis: Yueyue $\mathrm{Xu}$; Obtaining funding: Zhibing Qiu; Drafting the manuscript: Zhibing Qiu; Revision of manuscript for important intellectual content: Zhibing Qiu and Xin Chen.

\section{CONFLICTS OF INTEREST}

The authors have declared that no competing interests exist.

\section{FUNDING}

This study was supported by National Natural Science Foundation of China ( project no. 81270192) and Jiangsu Top Expert Program in Six Professions ( project no. 2014-WSW-052). 


\section{REFERENCES}

1. Elliott P, Andersson B, Arbustini E, Bilinska Z, Cecchi F, Charron P, Dubourg O, Kühl U, Maisch B, McKenna WJ. Classification of the cardiomyopathies: a position statement from the European Society Of Cardiology Working Group on Myocardial and Pericardial Diseases. Eur Heart J. 2007; 29:270-276.

2. Jefferies JL, Towbin JA. Dilated cardiomyopathy. Lancet. $2010 ; 375: 752-762$.

3. Adams KF, Dunlap SH, Sueta CA, Clarke SW, Patterson JH, Blauwet MB, Jensen LR, Tomasko L, Koch G. Relation between gender, etiology and survival in patients with symptomatic heart failure. J Am Coll Cardiol. 1996; 28:1781-1788.

4. Felker GM, Thompson RE, Hare JM, Hruban RH, Clemetson DE, Howard DL, Baughman KL, Kasper EK. Underlying causes and long-term survival in patients with initially unexplained cardiomyopathy. N Engl J Med. 2000; 342:1077-1084.

5. Mahon NG, Murphy RT, MacRae CA, Caforio AL, Elliott PM, McKenna WJ. Echocardiographic evaluation in asymptomatic relatives of patients with dilated cardiomyopathy reveals preclinical disease. Ann Intern Med. 2005; 143:108-115.

6. Lakdawala NK, Funke BH, Baxter S, Cirino AL, Roberts AE, Judge DP, Johnson N, Mendelsohn NJ, Morel C, Care $\mathrm{M}$. Genetic testing for dilated cardiomyopathy in clinical practice. J Cardiac Fail. 2012; 18:296-303.

7. Kataoka M, Wang DZ. Non-coding RNAs including miRNAs and lncRNAs in cardiovascular biology and disease. Cells. 2014; 3:883-898.

8. Ounzain S, Micheletti R, Beckmann T, Schroen B, Alexanian M, Pezzuto I, Crippa S, Nemir M, Sarre A, Johnson R. Genome-wide profiling of the cardiac transcriptome after myocardial infarction identifies novel heart-specific long non-coding RNAs. Eur Heart J. 2014; 36:353-368.

9. Kumarswamy R, Bauters C, Volkmann I, Maury F, Fetisch J, Holzmann A, Lemesle G, Pinet F, Thum T. The circulating long non-coding RNA LIPCAR predicts survival in heart failure patients. Circ Res. 2014; 114:1569-1575.

10. Michalik KM, You X, Manavski Y, Doddaballapur A, Zörnig M, Braun T, John D, Ponomareva Y, Chen W, Uchida S, Boon RA, Dimmeler S. The long noncoding RNA MALAT1 regulates endothelial cell function and vessel growth. Circ Res. 2014; 114:1389-1397.

11. Zhang Y, Zhang M, Xu W, Chen J, Zhou X. The long noncoding RNA H19 promotes cardiomyocyte apoptosis in dilated cardiomyopathy. Oncotarget. 2017; 8:28588. https:// doi.org/10.18632/oncotarget.15544.

12. Frade AF, Laugier L, Ferreira LR, Baron MA, Benvenuti LA, Teixeira PC, Navarro IC, Cabantous S, Ferreira FM, da Silva Cândido D, Gaiotto FA, Bacal F, Pomerantzeff $\mathrm{P}$, et al. Myocardial infarction-associated transcript, a long noncoding RNA, is overexpressed during dilated cardiomyopathy due to chronic Chagas disease. J Infect Dis. 2016; 214:161-165.

13. Thum T, Condorelli G. Long noncoding RNAs and microRNAs in cardiovascular pathophysiology. Circ Res. 2015; 116:751.

14. Beltrami C, Angelini TG, Emanueli C. Non-coding RNAs in diabetes vascular complications. J Mol Cell Cardiol. 2015; 89:42-50.

15. Greco S, Zaccagnini G, Perfetti A, Fuschi P, Valaperta R, Voellenkle C, Castelvecchio S, Gaetano C, Finato $\mathrm{N}$, Beltrami AP. Long noncoding RNA dysregulation in ischemic heart failure. J Transl Med. 2016; 14:1-14.

16. Frade AF, Laugier L, Ferreira LR, Baron MA, Benvenuti LA, Teixeira PC, Navarro IC, Cabantous S, Ferreira FM, da Silva Cândido D, Gaiotto FA, Bacal F, Pomerantzeff $\mathrm{P}$, et al. Myocardial infarction-associated transcript, a long noncoding RNA, is overexpressed during dilated cardiomyopathy due to chronic Chagas disease. Journal of Infectious Diseases. 2016; 214:161-165.

17. Merlet P, Caussin C, Poiseau E, Piot O, Maziere B, Syrota A. In vivo assessment of neurotransmitter system in cardiovascular diseases. Clinical issues. Q J Nucl Med. 1996; 40:108-120.

18. Qin F, Vulapalli RS, Stevens SY, Liang CS. Loss of cardiac sympathetic neurotransmitters in heart failure and NE infusion is associated with reduced NGF. Am J Physiol Heart Circ Physiol. 2002; 282:H363.

19. Reményi A, Lins K, Nissen LJ, Reinbold R, Schöler HR, Wilmanns M. Crystal structure of a POU/HMG/DNA ternary complex suggests differential assembly of Oct4 and Sox2 on two enhancers. Genes Dev. 2003; 17:2048-2059.

20. Wunderle VM, Critcher R, Ashworth A, Goodfellow PN. Cloning and characterization of SOX5, a new member of the human SOX gene family. Genomics. 1996; 36:354-358.

21. Pfeufer A, Van Noord C, Marciante KD, Arking DE, Larson MG, Smith AV, Tarasov KV, Müller M, Sotoodehnia N, Sinner MF. Genome-wide association study of PR interval. Nat Genet. 2010; 42:153-159.

22. Eijgelsheim M, Newton-Cheh C, Sotoodehnia N, de Bakker PI, Müller M, Morrison AC, Smith AV, Isaacs A, Sanna S, Dörr M. Genome-wide association analysis identifies multiple loci related to resting heart rate. Hum Mol Genet. 2010; 19:3885-3894.

23. Della-Morte D, Beecham A, Rundek T, Wang L, McClendon MS, Slifer S, Blanton SH, Di Tullio MR, Sacco RL. A follow-up study for left ventricular mass on chromosome 12p11 identifies potential candidate genes. BMC Med Genet. 2011; 12:100.

24. Olesen MS, Holst AG, Jabbari J, Nielsen JB, Christophersen IE, Sajadieh A, Haunsø S, Svendsen JH. Genetic loci on chromosomes 4q25, 7p31, and 12p12 are associated with onset of lone atrial fibrillation before the age of 40 years. Can J Cardiol. 2012; 28:191-195. 
25. Li A, Ahsen OO, Liu JJ, Du C, McKee ML, Yang Y, Wasco W, Newton-Cheh CH, O'Donnell CJ, Fujimoto JG. Silencing of the Drosophila ortholog of SOX5 in heart leads to cardiac dysfunction as detected by optical coherence tomography. Hum Mol Genet. 2013; 22:3798-3806.

26. Townsend-Nicholson A, Baker E, Schofield PR, Sutherland GR. Localization of the adenosine A1 receptor subtype gene (ADORA1) to chromosome 1q32.1. Genomics. 1995; 26:423-425.

27. Funaya H, Kitakaze M, Node K, Minamino T, Komamura $\mathrm{K}$, Hori M. Plasma adenosine levels increase in patients with chronic heart failure. Circulation. 1997; 95:1363-1365.

28. Xu X, Fassett J, Hu X, Zhu G, Lu Z, Li Y, Schnermann J, Bache RJ, Chen Y. Ecto-5'-nucleotidase deficiency exacerbates pressure-overload-induced left ventricular hypertrophy and dysfunction. Hypertension. 2008; 51:1557-1564.

29. Puhl SL, Kazakov A, Müller A, Fries P, Wagner D, Böhm M, Maack C, Devaux Y. Adenosine A1 receptor activation attenuates cardiac hypertrophy and fibrosis in response to $\alpha 1$-adrenoceptor stimulation in vivo. Br J Pharmacol. 2016; 173:88-102.

30. Lamb J, Crawford ED, Peck D, Modell JW, Blat IC, Wrobel MJ, Lerner J, Brunet JP, Subramanian A, Ross KN. The Connectivity Map: using gene-expression signatures to connect small molecules, genes, and disease. Science. 2006; 313:1929-1935.

31. Schmieder R, Edwards R. Quality control and preprocessing of metagenomic datasets. Bioinformatics. 2011; 27:863-864.

32. Trapnell C, Pachter L, Salzberg SL. TopHat: discovering splice junctions with RNA-Seq. Bioinformatics. 2009; 25:1105-1111.

33. Harrow J, Frankish A, Gonzalez JM, Tapanari E, Diekhans M, Kokocinski F, Aken BL, Barrell D, Zadissa A, Searle S. GENCODE: the reference human genome annotation for The ENCODE Project. Genome Res. 2012; 22:1760-1774.
34. Pertea M, Pertea GM, Antonescu CM, Chang TC, Mendell JT, Salzberg SL. StringTie enables improved reconstruction of a transcriptome from RNA-seq reads. Nat Biotechnol. 2015; 33:290-295.

35. Robinson MD, McCarthy DJ, Smyth GK. edgeR: a Bioconductor package for differential expression analysis of digital gene expression data. Bioinformatics. 2010; 26:139-140.

36. Langfelder $\mathrm{P}$, Horvath S. WGCNA: an R package for weighted correlation network analysis. BMC Bioinformatics. 2008; 9:559.

37. Yu G, Wang LG, Han Y, He QY. clusterProfiler: an R package for comparing biological themes among gene clusters. OMICS. 2012; 16:284-287.

38. Xiong Q, Ancona N, Hauser ER, Mukherjee S, Furey TS. Integrating genetic and gene expression evidence into genome-wide association analysis of gene sets. Genome Res. 2012; 22:386-397.

39. Shannon P, Markiel A, Ozier O, Baliga NS, Wang JT, Ramage D, Amin N, Schwikowski B, Ideker T. Cytoscape: a software environment for integrated models of biomolecular interaction networks. Genome Res. 2003; 13:2498-2504.

40. Szklarczyk D, Franceschini A, Wyder S, Forslund K, Heller D, Huerta-Cepas J, Simonovic M, Roth A, Santos A, Tsafou KP. STRING v10: protein-protein interaction networks, integrated over the tree of life. Nucleic Acids Res. 2014; 43:D447-D452.

41. Bader GD, Hogue CW. An automated method for finding molecular complexes in large protein interaction networks. BMC Bioinformatics. 2003; 4:2.

42. Lamb J, Crawford ED, Peck D, Modell JW, Blat IC, Wrobel MJ, Lerner J, Brunet JP, Subramanian A, Ross KN. The Connectivity Map: using gene-expression signatures to connect small molecules, genes, and disease. Science. 2006; 313:1929-1935. 